\title{
Chemical dissolution of carbonate rocks: A micro-CT study
}

\author{
Jafar Qajar ${ }^{1}$ and Christoph H. Arns ${ }^{2}$ \\ ${ }^{1}$ School of Chemical and Petroleum Engineering, Shiraz University, Shiraz, Iran \\ ${ }^{2}$ School of Petroleum Engineering, The University of New South Wales, Sydney, NSW 2052, Australia
}

The evolution of rock microstructure is a result of complex interactions between the rock surface and reactive fluids and depends on the thermodynamic conditions, the rock and fluid composition and the flow regime [1]. At the pore-scale, the dissolution mechanism consists of mass transport of the reactant by diffusion and advection to the solid surface, chemical reaction at the fluid-mineral interface, and the product mass transport away from the surface [2]. Natural heterogeneity of the porous rocks for the case of rapid dissolution of the mineral by the reactant fluid results in localization of the flow path and the formation of highly conductive channels called wormholes [3]. Several studies have revealed a variety of dissolution patterns from face dissolution to uniform dissolution depending on the injection rate and fluid/mineral properties [4-6].

A number of imaging techniques such as Wood's metal casting, neutron radiography and scanning electron microscopy have been used to study the dissolution patterns. Among them, X-ray micro-computed tomography (micro-CT) is a superior non-destructive imaging method which can create high-resolution images with large field of view [7-10].

In this work, two carbonate core samples were considered for the dissolution experiments. The first sample was an oolitic limestone with multi-modal pore size distribution, while the second one was a wackestone-packstone carbonate with a bimodal pore size. Dissolution experiments were conducted in core plugs of $7 \mathrm{~mm}$ diameter using ethylenediaminetetraacetic acid (EDTA) solution at $\mathrm{pH} 12$ as the reactive fluid. The experiments were performed at ambient conditions and were terminated after significant changes occurred in the cores as indicated by the pressure transducers. Details of the experimental procedure were described in [11].

The pre- and post-dissolution dry samples were imaged using a high resolution micro-CT scanner at the Australian National University (ANU) [12] with at least $8.5 \times 8.5 \times 8.5 \mathrm{~mm}$ field of view and resolutions of less than $5 \mu \mathrm{m}$. The post-dissolution image was superposed to the pre-dissolution image using a $3 \mathrm{D}$ registration technique developed by Latham, et al. [13]. In the micro-CT image, the grey-scale values of the pixels correspond to the density and composition of the material imaged. Hence, in monomineralic rock, such as the samples studied in this paper, micro-CT images are $3 \mathrm{D}$ porosity maps.

Figures 1 and 2 show examples of registered slices of the oolitic sample from top and side views, respectively. Visual observations of the images clearly illustrate that the reactive fluid locally increased the pore diameters across most parts of the sample and hence led to a quasi-uniform dissolution pattern. This pattern belongs to local nonequilibrium dissolution category [2] in which there is no sharp interface between the reactive fluid zone and the porous medium.

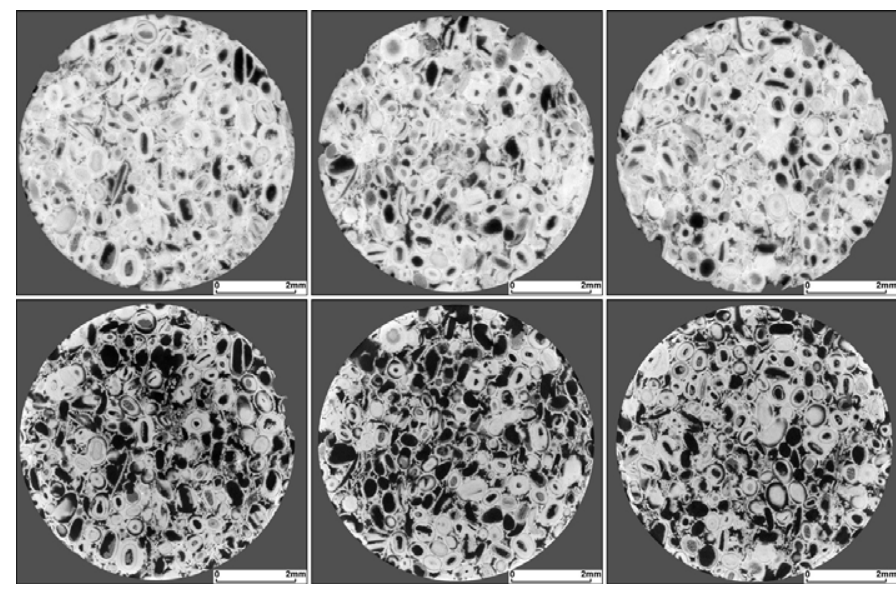

Figure 1. (Top) pre-dissolution micro-CT slices of the oolitic sample; (Bottom) the corresponding registered post-dissolution images. The slices are at different distances to the front (injection face) of the samples: (left) $6.42 \mathrm{~mm}$, (middle) $9.50 \mathrm{~mm}$, and (right) $11.55 \mathrm{~mm}$.

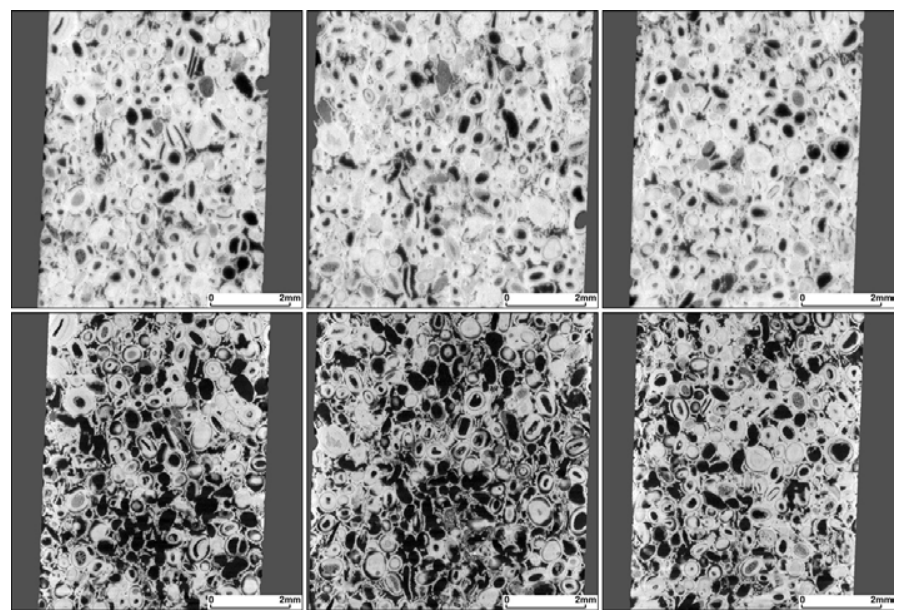

Figure 2. Side-view registered slices of the oolitic sample in (top) pre-dissolution and (bottom) post-dissolution states, at different positions.

Correspondence to: Jafar Qajar, School of Chemical and Petroleum Engineering, Shiraz University, Shiraz, Iran, Tel: +98-71-3613-3753, E-mail: jqajar@shirazu.ac.ir

Received: May 22, 2017; Accepted: June 09, 2017; Published: June 13, 2017 
Figures 3 and 4 illustrate examples of registered slices of the wackestone-packstone sample from top and side views, respectively. In contrary to the observation on the oolitic sample, visual observations of the images of the second sample reveal a different dissolution pattern which is called conical wormhole dissolution pattern. In this pattern, the reactive fluid was consumed over small parts of the mineral surface area leading to the formation of a few highly conductive flow channels, i.e. wormholes. The conical wormhole corresponds to the localequilibrium dissolution category [2] in which there is a sharp interface between the fluid zone and the medium as indicated in Figures 3 and 4 .
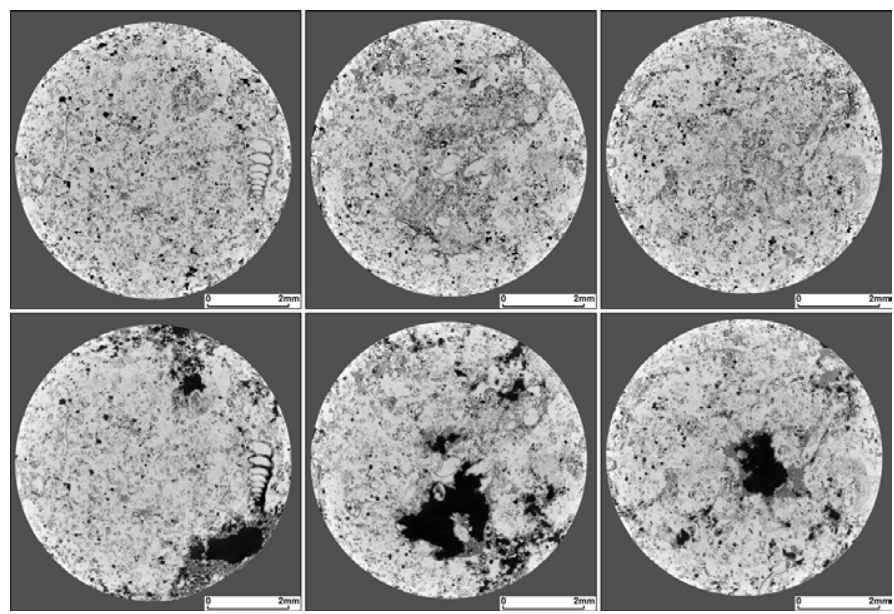

Figure 3. (Top) pre-dissolution micro-CT slices of the wackestone-packstone sample; (Bottom) the corresponding registered post-dissolution images. The slices are at different distances to the injection face of the samples: (left) $5.84 \mathrm{~mm}$, (middle) $7.98 \mathrm{~mm}$, and (right) $10.11 \mathrm{~mm}$.

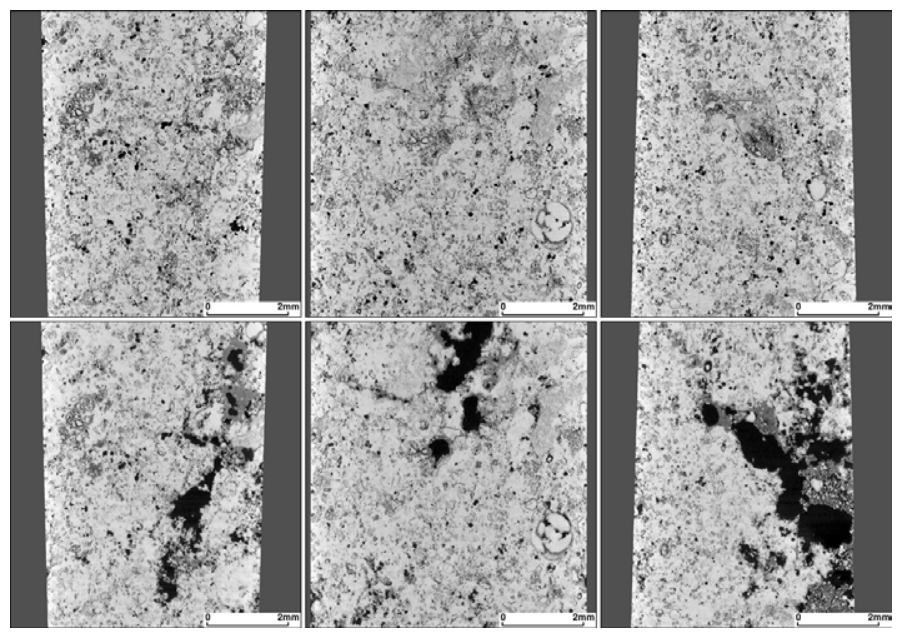

Figure 4. Side-view registered slices of the wackestone-packstone sample in (top) predissolution and (bottom) post-dissolution states, at different positions.
As found in this work, the micro-CT technique can provide high resolution images of successively disturbed specimens. This, in turn provides an important basis to calculate the evolution of rock microand macro-scale properties using advanced numerical techniques at the pore scale. In addition, micro-CT is a useful method to study pore scale reactive displacement mechanisms and their effects on the evolution of rock properties.

\section{References}

1. Algive L, Bekri S, Robin M, Vizika O (2007) Reactive transport: Experiments and porenetwork modelling, Paper SCA2007-10, presented at the International Symposium of the Society of Core Analysts. 10-12 September, Calgary, Canada.

2. Golfier F, Bazin B, Lenormand R, Quintard M (2004) Core-scale description of porous media dissolution during acid injection -Part I: Theoretical development. Computational and Applied Mathematics 23: 173-194.

3. Hoefner ML, Fogler HS (1988) Pore evolution and channel formation during flow and reaction in porous-media. AIChE Journal 34: 45-54.

4. Daccord G, Lietard O, Lenormand R (1993) Chemical dissolution of a porousmedium by a reactive fluid .2. Convection Vs Reaction, Behavior Diagram. Chemical Engineering Science 48: 179-186.

5. Fredd CN, Fogler HS (1998) Influence of transport and reaction on wormhole formation in porous media. AIChE Journal 44: 1933-1949.

6. Golfier F, Zarcone C, Bazin, B, Lenormand R, Lasseux D, Quintard M (2002) On the ability of a Darcy-scale model to capture wormhole formation during the dissolution of a porous medium. Journal of Fluid Mechanics 457: 213-254.

7. Spanne P, Thovert JF, Jacquin CJ, Lindquist WB, Jones KW, Adler PM (1994) Synchrotron computed microtomography of porous media: Topology and transports. Physical Review Letters 73: 2001-2004.

8. Shah SM, Gray F, Crawshaw JP, Boek ES (2015) Micro-computed tomography porescale study of flow in porous media: Effect of voxel resolution. Advances in Water Resources.

9. Sheppard AR, Sok RM, Averdunk H, Robins VB, Ghous A (2006) Analysis of Rock Microstructure Using High Resolution X-ray Tomography, Paper SCA2006-26, presented at the International Symposium of the Society of Core Analysts. 12-16 September 2006, Trondheim, Norway.

10. Knackstedt MA, Golab A, Carnerup A, Senden T, Butcher A, Riepe L (2011) Multiscale Formation Evaluation of Tight Gas Resources, Paper IPTC 14919-MS, presented at the International Petroleum Technology Conference, Society of Petroleum Engineers. International Petroleum Technology Conference, Bangkok, Thailand.

11. Qajar J (2012) Reactive flow in carbonate cores via digital core analysis, PhD Thesis, University of New South Wales.

12. Sakellariou A, Senden TJ, Sawkins TJ, Knackstedt MA, Turner ML, et al.(2004) An $\mathrm{x}$-ray tomography facility for quantitative prediction of mechanical and transport properties in geological, biological and synthetic systems., Developments in X-Ray Tomography IV, pp. 473-484.

13. Latham S, Varslot T, Sheppard AP (2008) Image registration: Enhancing and calibrating X-ray micro-CT imaging, Paper SCA2008-35, presented at the International Symposium of the Society of Core Analysts. 29 October-2 November 2008, Abu Dhabi, UAE.

Copyright: (C2017 Qajar J. This is an open-access article distributed under the terms of the Creative Commons Attribution License, which permits unrestricted use, distribution, and reproduction in any medium, provided the original author and source are credited. 